\title{
Reliable Smart Techniques in Healthcare: A Descriptive Study on Developing IoMT Based System Model
}

\author{
R. Jegan ${ }^{1}$, P. Subha Hency Jose ${ }^{2}$, Sweety Jose ${ }^{3}$, Nimi W.S ${ }^{4}$ \\ $\left\{\right.$ jegan@karunya.edu ${ }^{1}$, hency20002000@karunya.edu ${ }^{2}$, psj.eee@psgtech.ac.in ${ }^{3}$, \\ nimiwsrec@gmail.com ${ }^{4}$ \} \\ ${ }^{1,2,4}$ Department of Biomedical Engineering, Karunya Institute of Technology and Sciences \\ ${ }^{3}$ Department of EEE, PSG College of Technology, Coimbatore, India
}

\begin{abstract}
The primary objective of this paper is to review the current impact of IoMT (Internet of Medical Things) and their implementation in healthcare networks. A detailed study was made regarding the emerging technologies which can be applied in the healthcare for monitoring patient health conditions and diagnosing various diseases. The different research papers related with IoMT were collected and reviewed. This paper proposed the possible emerging trends, methodologies and applications of different smart techniques which can be involved in improving the efficiency of continuous monitoring, diagnosis and treatment. In particular, the treatment of infected person who are in critical stage is a big headache as they are to be kept in ventilators and need timely care. As the number of infected cases increases, many countries are struggling to give proper care which results in death of patient. The proposed techniques play a main role and help the medical providers to give a timely support to the infected patients. The proposed techniques can be used for improving the patient care with automatic observations.
\end{abstract}

Keywords: RFID, component, formatting, style, styling, insert.

\section{Introduction}

Real time continuous monitoring of patient's health condition has great potential to save many individual life. Patient data monitoring is a key issue for biomedical research. This paper proposes a key techniques to design a smart biomedical system with IoMT concepts in healthcare network for solving human problems during pandemic situations. It also describes the state of art design criteria, different methodologies and various applications of IoMT in healthcare network. Currently, the spread of corona virus is growing very fast and the number of infectedpatients is rapidly increasing everydayin India. However various manufacturers are doing research to finda solution tocure at the earliest. Due to increasing number of infected patients, providing timely treatment and monitoring of the patients in healthcare network becomes challenging. It is necessary to bring additional emerging technologies which can be applicable for treating patient in very effective manner in healthcare network. This paper aims to highlight the applicable emerging technologies such as Internet of Medical Things (IoMT) with integration of Robotics, Artificial Intelligence, 
Cloud computing, Big data analytics, Sensor technology and Virtual reality to provide a valuable support to the society. The relevant information from the scientific environment has been reviewed and proposed useful system using these technologies in healthcare network. The dynamic role of IoMT becomes one

of the modern technologies in healthcare environment to solve many human problems[1]. The report says that implementation of IoT in various domains will reach 75 billion by the end of 2025 [2].The integration of wireless communication technology, sensor interfacing and cloud computing could develop a new working platform to sense the data, process it and takes the decision automatically. Nowadays, applications of Internet of things are drastically increasing in many scientific domains due to its ability to transmit the data and security. The implementation of IoT in healthcare provides an efficient way to monitor patient health conditions. The physiological parameters such as heart rate, pulse rate, $\mathrm{SpO} 2$, blood pressure, blood glucose level, respiration rate which are key vital parameters for analyzing patient health status. Therefore, it is very much essential to develop real time biomedical measurement system with IoMT technologies to sense the patient signal and enable continuous monitoring.

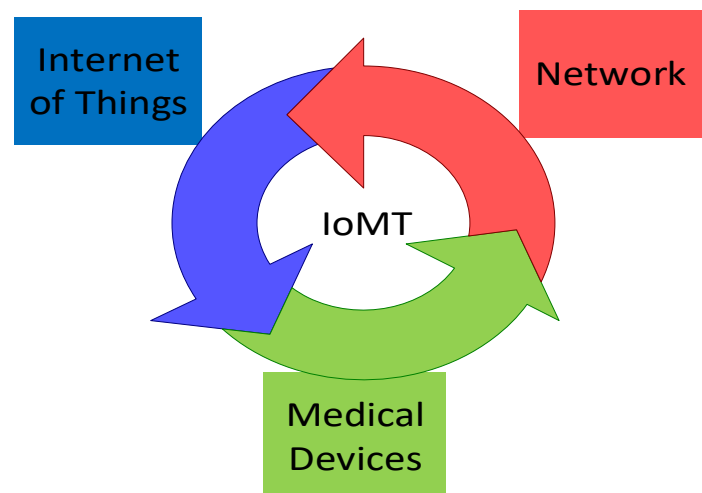

Figure 1: Structure of IoMT in healthcare network

The technical contents in this paper are divided into 5 sections. First section of this paper provides the detail information about the need and significance of IoMT in healthcare environment. Second section highlights the role of biosensors and their importance to monitor the vital parameters. Third section summarizes the architecture of IoMT and its various components involved for healthcare applications. Fourth section provides the implementation techniques with different wireless modules. Fifth section of this paper recommends the design consideration, future direction for developing an innovative healthcare application.

\section{Need And Significance Of Iomt In Healthcare Environment}

Internet of Medical Things (IoMT) brings every information at various interconnected devices to the internet. Recently, the need forIoMT around the world in biomedical environment becomes very essential because of online monitoring, efficient understanding of patient data, provide timely health information to the patient and remote support. The main impact of COVID-19 has dynamically spread across the world and affected many humans. At 
this time, medical providers in healthcare network have done a great work to treat the infected patient, but the spreading of virus among human population is drastically increasing. This has created major issues such as insufficient place, lack of manpower and providing individual treatment, loss of continuous monitoring. Therefore, it is very necessary to implement IoMT in healthcare network to fight with COVID-19 [3]. The role of IoMT is to collect the patient data such as heart rate, respiration rate, blood pressure, $\mathrm{SpO} 2$ and needed vital parameters through the sensors attached with patient. The development of IoMT based biomedical system includes four main modules: Signal Acquisition, Pre-processing, Feature Extraction and Integration with an Internet service. The data collected from sensors are transmitted to the cloud through internet and can be visualize by medical care provider. The advantage of using IoMT is to reduce time to provide an efficient treatment, continuously monitoring the status ofpatient and analyzing their health in remote. This methodology can save the life of many patients and also healthcare providers.

\section{Architecture Of IOMT}

In current situation, the methods integrated with emerging technologies helps the society for controlling and monitoring the spread of virus effectively. The implementation of proposed methodologies in healthcare network brings an alternative solution to the human society in terms of life saving measures, continuous monitoring of patient stress level, treatment and tracking the spread of virus [4]. The concepts proposed by various listed technologies are very flexible to implement in hospital environment and also support the medical providers to treat the patients effectively. This section of this paper focuses on the use of technologies such as IoMT, AI, virtual reality, robots, cloud computing, big data and biosensor technologies in healthcare sector. The implementation of proposed technologies in healthcare network in view of COVID-19 pandemic is shown in Figure2.

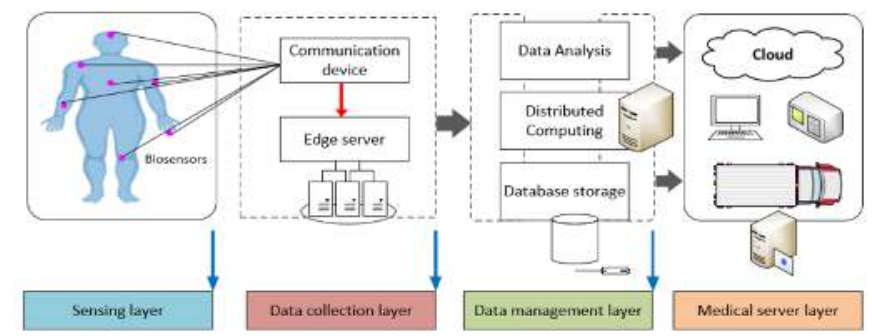

Figure 2: Graphical diagram depicting the structure of IoMT system

The architecture of IoMT system mainly consists of four layers. The primary importance of sensing layer provides the necessary sensing of patient vital signs. Here, various sensing modules play a vital role for acquiring real time signal. The data collection has the ability to receive the data from sensor and edge server to distribute the data to data management layer. In data management layer, the received informations are properly arranged for better analysis and stored as a database. Finally, the analysed data will be communicated from data management layer to cloud medical server for online monitoring with the support of cloud and internet based technologies. 


\section{Proposed System Model}

Various smart technologies presented in this paper provides an emerging phenomena for enabling the system to montor human vital parameters continuously in healthcare environment. The integration of these technologies offers wide range of innovative applications in biomedical engineering with grat potential support to patient dailylife activities. The proposed system model which offers continuous monitoring with smart technologies is shown in Figure 3.

\section{Role Of Biosensors And Their Importance}

Nowadays the concept of wearable sensor has a dynamic role in healthcare network because of long-term continuous acquisition and wearability[5-10]. The advances in sensing technology with innovative algorithms have provided an efficient design of sensor based medical measurement systems [11-12]. Portable wearable devices are essential in today's world situation for various applications such as monitoring patient health, identifying and isolation [13]. According to the data published, the major symptoms of COVID-19 infected patients are fever, cough and respiration problems. Additionally, the patient also experienced headache, reducing taste and muscle pain. Therefore, proper diagnosing the presence of this novel coronavirus helps the society to control its spreading among human populations. The role of wearable bio sensing is to sense the basic symptoms such as fever, muscle pain and respiration rate through sensors. 


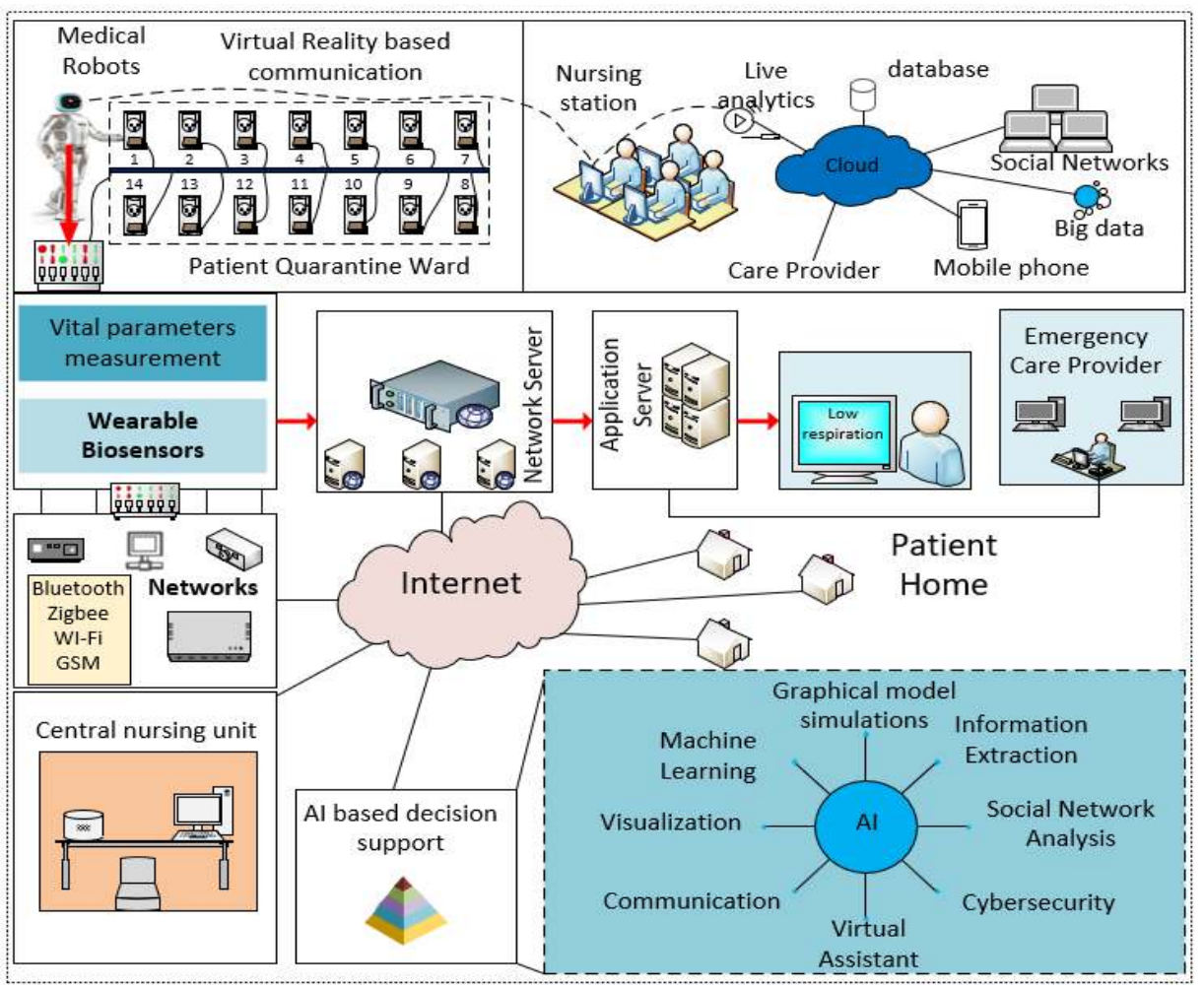

Figure 3: Integration of Smart technologies in healthcare network

The rapid development of various forms of biosensors with advanced learning algorithms helps the medical workers to recognize many health issues [14]. The function of this biosensor provides accurate measurement of body biological and chemical substances. Additionally, the role and use of ingestible sensors during COVID-19 becomes very effective methods which can provide the health status of internal organ of the body. These sensors can be connected to internet for remote monitoring. This will help the medical providers to assist patient health both internally and externallyand also control the diseases among hospital environments. There are many number of biosensors are used in biomedical applications. Figure 4 provides the detailed view of different group of biosensors. The most widely used sensor in healthcare domains are categorized as the source of measurement mediums such as using bio receptors and transducer. Mostly, bioelectric sensor can be used to develop a non-invasive device which offers the integration of electronic circuits with biological elements. The conversion of chemical activity into an electrical signal can be done with the help of biochemical sensor. The applicable use of biosensor in healthcare network is shown in Figure 5. 


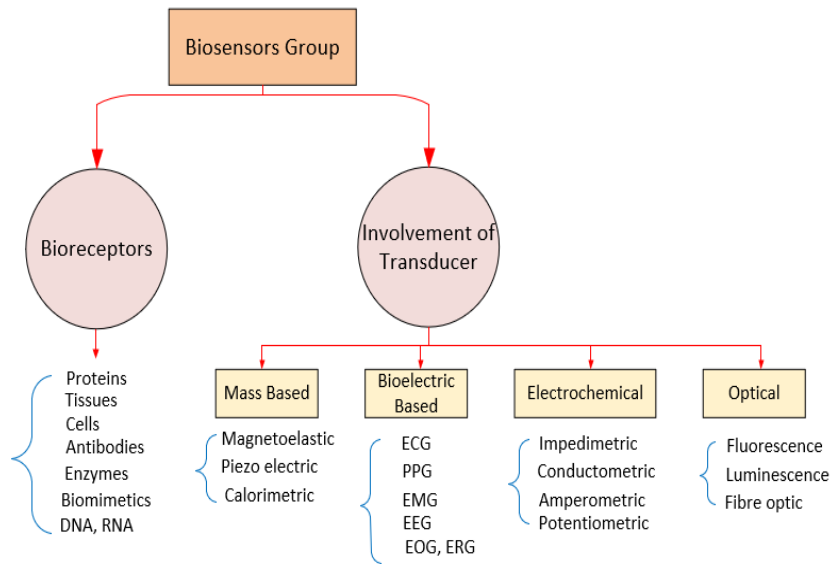

Figure 4: Group of Biosensors in healthcare network

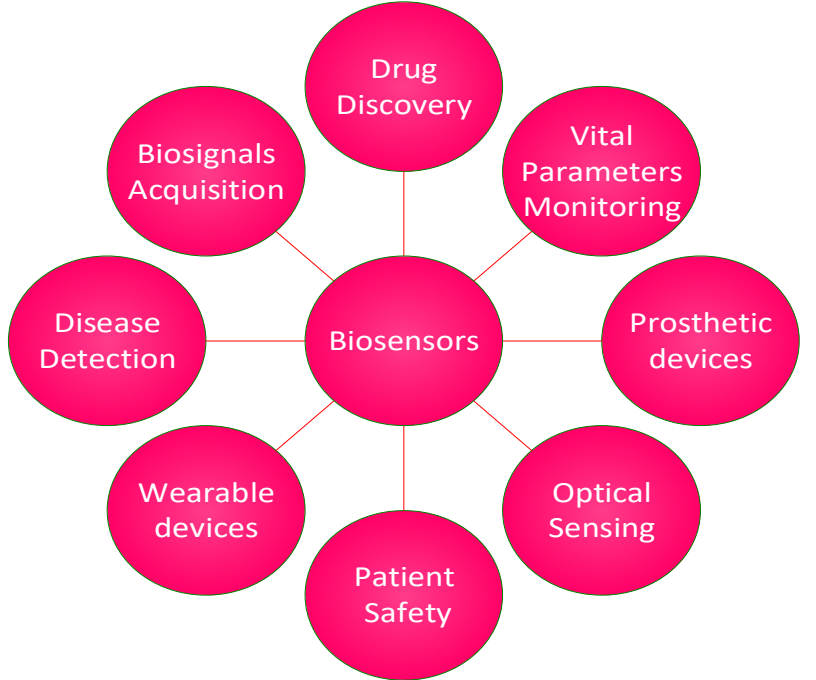

Figure 5: Role of Biosensors in healthcare network

In many cases, Electrocardiogram (ECG), Photoplethysmogram (PPG) and Electroencephalogram (EEG) sensors provides an efficient representation of biomedical signals which are very helpful for immediate analysis of patient health conditions. The required vital parameters can be easily extracted from the acquired signals which includes heart rate, pulse rate, respiration rate, heart rate variability, mental stress, brain disorders and also analyzing various cardiac arrhythmias. The acquired signal from these sensors and its noise removed signals are shown in Figures $6,7 \& 8$. 


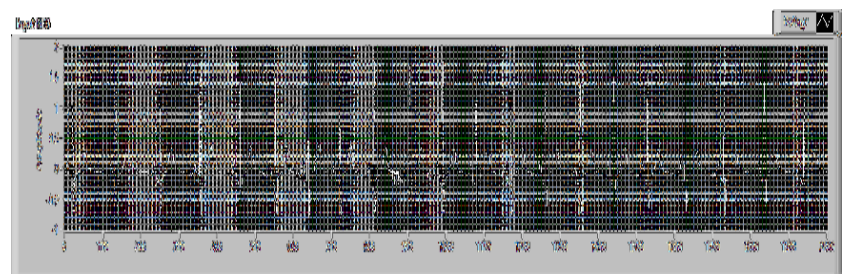

Figure 6: Representation of ECG signal acquired from ECG sensor attached to human body

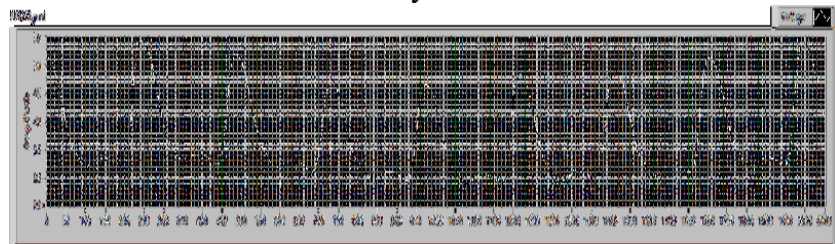

Figure 7: Representation of PPG signal acquired from PPG sensor attached to human body

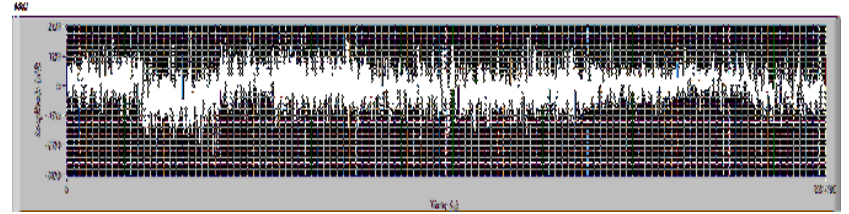

Figure 8: Representation of EEG signal acquired from EEG electrodes attached to human body

\section{Medical Robotics}

In current situations, most of the healthcare providers provide a treatment to COVID19 patients in very effective manner. The respiratory droplets and personal contacts with affected person are main source for transmission of this virus [15-23]. The initial assessment of COVID-19 is to check the body temperature and provide a guideline to the patient for further testing. It is very difficult to provide an efficient treatment in healthcare services because of increasing number of patients. The use of wide range of medical robots with adaptive collision detection algorithm, roadmap motion planning, fastron algorithm, robot learning, reinforcement goal-oriented approach, and advanced machine vision and registration algorithms in healthcare network provides an alternative solution and is being increased to serve many roles in hospital environment. The medical robots can be used for collecting all necessary information from patient such as temperature, respiration rate and other needed data from the patient. This will avoid the need of nurse to collect the data to monitor the patient health. The medical providers can use these robots to provide a basic treatment in rural or remote locations. This solution has many advantages such as controlling spread of virus from infected patient to nurse and reduce workers stress level, provide large number of initial testing, long term monitoring without human intervention etc. 


\section{AI, BIG Data Analytics And Cloud Computing}

In healthcare network, there is a need for developing and utilizing new technologies to control and monitor the effect of many diseases. Recently, the world is facing a very critical situation by means of spreading the novel coronavirus and looking for some emerging technologies to support and control the spread of this virus. The role of AI (Artificial Intelligence) play a vital role in medical environment which includes identifying infected patients, prediction, monitoring the number of infections with accurate values by traning the system models [24].

The ideal role of classification algorithms, regression algorithms, clustering algorithms, ensemble learning algorithms in the field of AI becomes very effective technology in healthcare network in many ways. The instrument for treating COVID-19 need to be designed with the help of these algorithms by the manufacturer to provide better response. For example, identifying and confirming diseases by devices requires an efficient methodology to detect the correct medium of interest [20]. This can be done very effectively by an artificial intelligence technology [25]. The big data analytics provides analytical measures of all real time data for taking better decisions around the world by healthcare workers [26-27]. The most leading data science algorithms such as K-Means Clustering Algorithm, Ada-Boost, Association Rule Mining Algorithm, Linear Regression Algorithms, Logistic Regression Algorithms, C4.5 based decisions tree, Support Vector Machine (SVM), Apriori Algorithm, ExpectationMaximization Clustering Algorithms can be used to bring different data analysis through statistical approach by accurate classification, matching and predicting. Another efficient technology to fight with COVID-19 is cloud computing which can offer flexible services over the internet such as security mechanism of patient data, confidentiality of the data, recycling of data, data integrity, data transmission and cloud management. The advantages of this technology include accurate early detection, reduced work time for offline processing, decision making, tracking of infected patients, drug design and provides well accurate data about COVID-19.

\section{Virtual Reality}

Currently, the most significant control method used by all countries is social distancing. The rapid monitoring of viral infected patients and isolating them is one of the most safety measures to prevent the spread of this virus. Many countries introduced the concept of lockdown in their own region based on the effects of this virus. During this time, people are failing to complete their tasks in terms of their absence. The concept of virtual reality brings a solution to make people available to the environment without travelling [28]. By using this technique, people can interact with the model through optimization techniques. These provide an efficient mechanism to the hospital workers for proper communication and also enable them to deliver their task without any delay. This technology act as a leading role in the society by providing proper medical training, COVID-19 awareness, prevention steps. 


\section{Wireless Communication Technology And Monitoring}

Recently, the importance of using wireless modules in various applications are increasing due to the ability of transmitting data remotely. It will provide an effective monitoring system in which patient or care taker can visualize their data at any time. There are different wireless devices are currently used by many researchers in healthcare domains [29]. The below Table 1 summarize the possible wireless devices and their uses and application areas.

Table 1: Summary of application areas of wireless communication technology

\begin{tabular}{|l|l|}
\hline Technology & Application uses \\
\hline Wi-Fi & $\begin{array}{l}\text { Patient monitoring system, } \\
\text { Transmitting patient data }\end{array}$ \\
\hline Bluetooth & $\begin{array}{l}\text { Short range communication, } \\
\text { Patient monitoring system }\end{array}$ \\
\hline Li-Fi & $\begin{array}{l}\text { Healthcare, education, retail, } \\
\text { aviation, exhibition industry }\end{array}$ \\
\hline IrDA & $\begin{array}{l}\text { Wireless communication and } \\
\text { data transmission }\end{array}$ \\
\hline NFC & $\begin{array}{l}\text { Data transmission, Patient } \\
\text { monitoring system }\end{array}$ \\
\hline Broadcast Radio & $\begin{array}{l}\text { Wireless communication and } \\
\text { data transmission }\end{array}$ \\
\hline $\begin{array}{l}\text { Microwave } \\
\text { Communication }\end{array}$ & $\begin{array}{l}\text { Wireless communication and } \\
\text { data transmission }\end{array}$ \\
\hline
\end{tabular}

Table 2: Performance summary different wireless communication devices used in healthcare network

\begin{tabular}{|c|c|c|c|c|c|c|}
\hline Devices & Zigbee & BLE & Wi-Fi & GPRS & LoRa & SigFox \\
\hline $\begin{array}{l}\text { Standard } \\
\text { (IEEE) }\end{array}$ & 802.15 .4 & 802.15 .1 & $802.11 \mathrm{a}$ & NA & $802.15 .4 \mathrm{~g}$ & $802.15 .4 \mathrm{~g}$ \\
\hline $\begin{array}{l}\text { Frequency } \\
(\mathrm{MHz})\end{array}$ & $\begin{array}{l}868 / 915 \mathrm{an} \\
\text { d 2.4GHz }\end{array}$ & $2.4 \mathrm{GHz}$ & $2.4 \mathrm{GHz}$ & $900-1800$ & $869 / 915$ & $869 / 915$ \\
\hline Modulation & BPSK & GMSK & BPSK & GMSK & GPSK & GPSK \\
\hline RF Channel & $1,10,16$ & 40 & 11 & 124 & 10,8 & 360 \\
\hline Bandwidth & $2 \mathrm{MHz}$ & $1 \mathrm{MHz}$ & $22 \mathrm{MHz}$ & $200 \mathrm{KHz}$ & $<500 \mathrm{KHz}$ & $<100 \mathrm{~Hz}$ \\
\hline $\begin{array}{l}\text { Power } \\
\text { consumption }\end{array}$ & Low & Ultra Low & High & Medium & Low & Low \\
\hline Data rate & $\begin{array}{l}20,40,250 \\
\text { kbps }\end{array}$ & $1 \mathrm{Mbps}$ & $\begin{array}{ll}11-54, & 150 \\
\text { Mbps } & \\
\end{array}$ & Upto 170kbps & $50 \mathrm{kbps}$ & 100bps \\
\hline Latency & $20-30 \mathrm{~ms}$ & $6 \mathrm{~ms}$ & $50 \mathrm{~ms}$ & $<1 \mathrm{~ms}$ & NA & NA \\
\hline Range & $100 \mathrm{~m}$ & $10 \mathrm{~m}$ & $100 \mathrm{~m}$ & $1-10 \mathrm{Km}$ & $5 \mathrm{Km}$ & $10 \mathrm{Km}$ \\
\hline Network size & 65000 & - & 32 & 1000 & $\begin{array}{l}10000 \\
\text { (per BS) }\end{array}$ & $\begin{array}{l}1000000 \quad \text { (per } \\
\text { BS) }\end{array}$ \\
\hline Cost & Low & Low & High & Medium & Low & Low \\
\hline Application & Healthcare & Healthcare & Healthcare & Healthcare & Healthcare & Healthcare \\
\hline
\end{tabular}




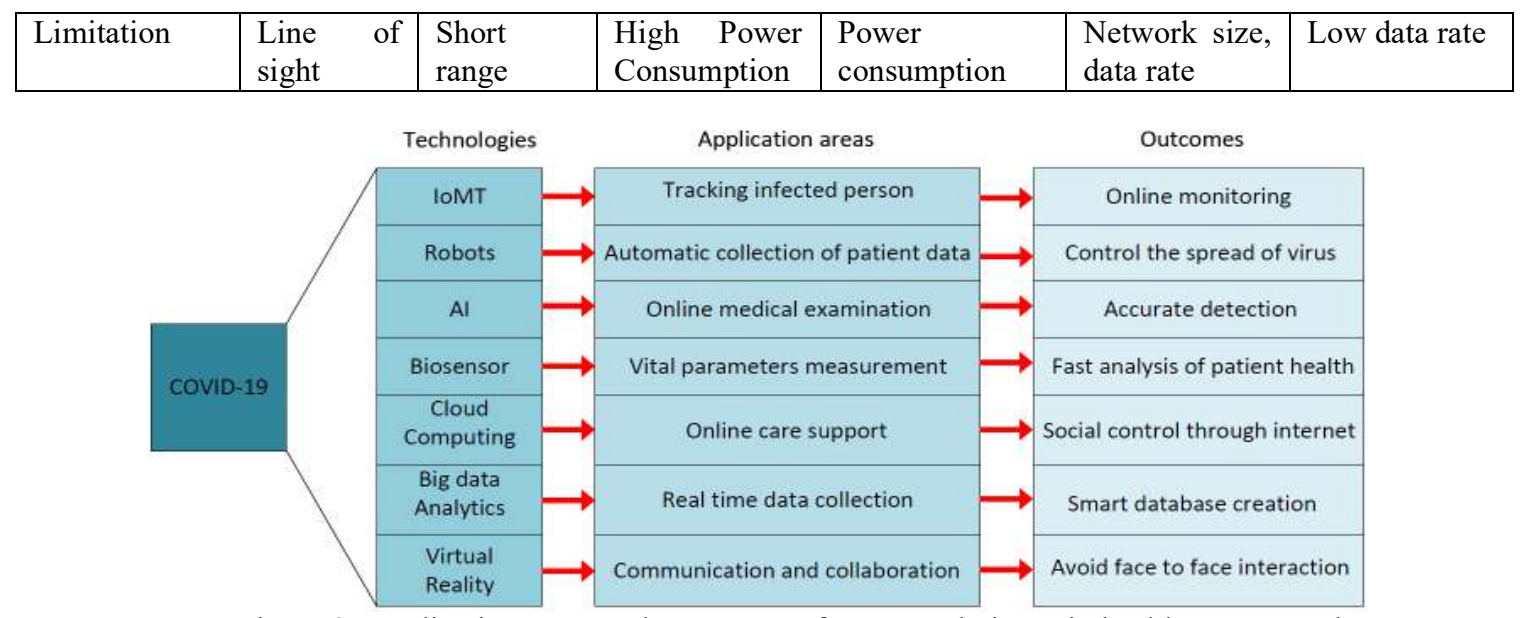

Figure 9: Application areas and Outcomes of Smart techniques in healthcare network

The integration of above wireless communication technology with wireless device has a flexibility to transmit the data remotely [30-33]. This can be easily interfaced with the sensing module for transmission of patient data.

\section{Conclusion And Future Directions}

The wearable sensing module play a main role in the proposed system. The devices consists of biomedical sensor which are attached to patient for collecting patient's real time data and measure the vital parameters. The design of wearable devices becomes one of most important challenging task among the researchers. The various key points such as low power consumption, energy harvesting, in built preprocessing unit, rapid monitoring by wireless devices and quality signal acquisition are considered as necessary design criteria for developing wearable devices. The advantages of wearable devices includes low cost, small size, patient comfort, without disturbing patient activities, life time and continuous monitoring. The different smart technology listed in this paper are very useful to develop a smart medical instrumentation system for e- healthcare applications. The proposed methodology can be applicable and considered for identifying the infected patients and monitoring their health condition remotely during the pandemic situations. The potential application areas and outcomes of these technologies on COVID-19 is shown in Figure 9. This paper addresses the detailed view of applicable technologies with IoMT concept for developing smart medical instrumentation system. This study mainly helps the developers and manufacturers to design a prototype model which can focus on providing efficient support to patient. In view of pandemic situations, it is very essential to focus on alternate engineering technology and their integration with medical sensors. The wide range of information presented in this paper focused on possible implementation of different smart technology in healthcare network. The networking concept and internet technology provide a flexibility to interface the sensing module to the cloud (Connected devices "IoT. The different network protocols can be used by researchers to communicate sensor data to the cloud platform for 
displaying patient data. This will support the patient by providing timely treatment and also lead to early detection of many diseases. During the wide spread of corona virus, the proposed system with smart technologies helps the society in great manner by providing technology support to the healthcare providers to solve human health related problems. From the detailed description of different technology given in this paper, it was concluded that the presented wireless communication technology with the integration of biosensors, medical robotics, virtual reality, artificial intelligence, big data and cloud computing technology can be suitable alternative to develop an innovative measurement system for many e-healthcare applications.

\section{References}

[1] Ravi Pratap Singh, MohdJavaid, AbidHaleem, RajuVaishya\&Shokat Ali. (2020). Internet of Medical Things (IoMT) for orthopaedic in COVID-19 pandemic: Roles, challenges, and applications. Journal of Clinical Orthopaedics and Trauma (press), 11(4):713-717.

[2] C.D "IoT: number of connected devices worldwide 2015-2025 |Statista," Statista. [Online].Available: https://www.statista.com/statistics/471264/iot-number-of-connected-devicesworldwide/. [Accessed: 21-06-2021].

[3] Usman M, Jan MA, He X \& Chen J. (2019). P2DCA: a privacy-preserving-based data collection and analysis framework for IoMT applications. IEEE J Sel Area Commun, 37(6), 1222-1230.

[4] Jegan, R., Ashwin, V.H., Rajalakshmy\& P., Jose. (2020). EEG based internal stress observa-tion: An overview of methods involved from EEG acquisition to system design. Journal of Critical Reviews, 7(9), 947-952

[5] Arthi, R.Jegan. (2014). Embedded Based Patient's Vital Sign Monitoring System using Wireless Methods.International Journal of Engineering Research \& Technology, 3(4), 835-837.

[6] R.Jegan\&K.V.Anusuya. (2015). Real-time ECG peak detection for heart rate measurement using wavelet packet transform. Int. J. Biomedical Engineering and Technology, 19(3), 244-254.

[7] R.Jegan\&K.V.Anusuya (2015). Design of Adaptive Noise Canceller for Portable Electrocardiogram Signal Monitoring System Based on Sensors.Sensor Letter, 13, 1061-1066.

[8] R.Jegan\&Nimi WS. (2017). Low cost and Improved performance measures on filtering techniques for ECG signal processing and TCP/IP based monitoring using LabVIEW. IEEE 4th Inter-national Conference on Advanced computing and Communication systems, 1-7.

[9] R Jegan\& WS Nimi. (2018). Sensor Based Smart Real Time Monitoring of Patients conditions Using Wireless Protocol. International Journal of E-Health and Medical Communications (IJEHMC), 9(3).

[10] R.Jegan\&K.V.Anusuya. (2018). Bio Sensor based Feature Extraction and physiological parameters measurement for Bio Medical applications. Int. J. Biomedical Engineering and Technology, 28(1).

[11] R.Jegan\&K.V.Anusuya. (2013). High-performance ECG signal acquisition for heart rate measurement. Int. J. Biomedical Engineering and Technology, 12(4), 371-381.

[12] Karthikeyan, Satheesh Kumar \&R.Jegan. (2014). Design and development of low-cost blood serum analyser using adaptive neuro fuzzy inference system. Int. J. Biomedical Engineering and Technology, 15(3), 261-272.

[13] Pejcic B, De Marco R \& Parkinson G. (2006). The role of biosensors in the detection of emerging infectious diseases.Analyst [Internet], 131(10), 1079-1090

[14] Rajvikram Madurai Elavarasan\& Rishi Pugazhendhi. (2020). Restructured society and environment: A review on potential technological strategies to control the COVID-19 pandemic. Science of the Total Environment, 725, 138858.

[15] Weiss S.R. \&Leibowitz J.L. (2011). Coronavirus pathogenesis.Adv Virus Res, 81, 85-164 .

[16] Erika Sifuentes Rodriguez. Deborah Palacios-Reyes. (2020). Covid-19: The outbreak caused by a new coronavirus, Bol Med Hosp Infant Mex, 77 (2): 47-53.

[17] Chih Cheng Lai, Tzu-Ping Shih, Wen-ChienKo, Hung-Jen Tang \& Po-RenHsueh. (2020). Severe acute respiratory syndrome coronavirus 2 (SARS-COV-2) and coronavirus disease-2019 (COVID- 
19): The epidemic and the challenges. International Journal of An-timicrobial Agents, 55 (3), 105924.

[18] Alexandra L. Phelan, Rebecca Katz \& Lawrence O. Gostin. (2020). The Novel Coronavirus originat-ing in Wuhan, China Challenges for Global Health Governance.Journal of the American Medical Association. 323(8), 709-710.

[19] [C. Amuthadevi, D. S. Vijayan, Varatharajan Ramachandran, "Development of air quality monitoring (AQM) models using different machine learning approaches", Journal of Ambient Intelligence and Humanized Computing, https://doi.org/10.1007/s12652-020-02724-2

[20] Yan RongGua, Quing Dong Cao \&Zhong Si Hong. (2020). The origin transmission and clinical therapies on coronavirus disease 2019(COVID-19) outbreak- an update on the status. Military Medical Research, 7(1), 1-10.

[21] Muhammad Adnan Shereen, Suliman Khan, AbeerKazmi, Nadia Bashir \&RabeeaSiddique. (2020). COVID-19 infection: Origin, transmission, and characteristics of human coronaviruses. Journal of Advanced Research, 24, 91-98.

[22] C. Huang, Y. Wang, X. Li, L. Ren, J. Zhao \& Y. Hu. (2020). Clinical features of patients infected with 2019 novel coronavirus in Wuhan, China, Lancet, 395: 497-506.

[23] Guan WJ, Ni ZY, Hu Y, Liang WH, Ou CQ \&He JX. (2020). Clinical characteris-tics of 2019 novel coronavirus infection in china.NEngl J Med.,110, 193-204.

[24] Nguyen, Thanh. (2020). Artificial Intelligence in the Battle against Coronavirus (COVID-19): A Survey and Future Research Directions. doi: 10.13140/RG.2.2.36491.23846 .

[25] Buch, V.H., Ahmed, I \&Maruthappu, M. (2018). Artificial intelligence in medicine: current trends and future possibilities. Br. J. Gen. Pract. 68, 143-144.

[26] Bates D.W, Saria S, Ohno-Machado L, Shah A \&Escoba, G. (2014). Big data in health care: using analytics to identify and manage high-risk and high-cost patients. Health Aff, 33, 1123-1131.

[27] Manogaran G, Thota C, Lopez D \&Sundarasekar R. (2017). Big data security intelligence for healthcare industry 4.0.Cyber security for Industry, 4.0. Cham: Springer; 103-126.

[28] Ravi Pratap Singh, MohdJavaid, RavinderKataria, MohitTyagi, AbidHaleem\& Rajiv Su-man. (2020). Significant applications of virtual reality for COVID-19 pandemic.Diabetes\& Met-abolic Syndrome: Clinical Research \& Reviews, 14: 661- 664

[29] Javaid M \&Haleem A. (2019). Industry 4.0 applications in medical field: a brief review. Curr. Med. Res. Pract, 9(3), 102-109.

[30] Aishwarya Kumar, Puneet Kumar Gupta \&AnkitaSrivastava.(2020). A review of modern technologies for tackling COVID-19 pandemic.Diabetes\& Metabolic Syndrome: Clinical Re-search \& Reviews, 14, 569-573

[31] MohdJavaid, AbidHaleem, RajuVaishya, ShashiBahl, Rajiv Suman\&AbhishekVaish. (2020). Industry 4.0 technologies and their applications in fighting COVID-19 pandemic.Diabetes\& Metabolic Syndrome: Clinical Research \& Reviews, 14, 419-422.

[32] R.Jegan\&K.V.Anusuya. (2015). Heart Signal Monitoring For Remote Patient Assistant Based On Optical Remote Sensing.Optoelectronics and Advanced Materials - Rapid Communications 9(5), $570-574$.

[33] R.Jegan, Nimi\&JinoRamson. (2020). Sensor based biomedical frame work for monitoring patient vital parameters. IEEE International conference on Circuits and Systems, 96-100. 